\title{
Sustained Need for High-Dose Zinc Supplementation in Children With Acrodermatitis Enteropathica
}

\author{
Johanna Hammersen, MD', Cristina Has, $\mathbf{M D}^{2}$, Matthias Galiano, MD', \\ Martin Lindner, MD $^{3}$, Rainer Rossi, MD $^{4}$, Jürgen Kohlhase, MD $^{5}$, \\ and Holm Schneider, MD'
}

\section{Introduction}

Acrodermatitis enteropathica (AE) is a recessively inherited defect of intestinal zinc absorption with an estimated incidence of $1 / 500000 .^{1}$ The affected zinc transporter protein, ZIP4, is encoded by the gene SLC 39A4. ${ }^{2}$ As cofactor for many metalloenzymes and as a component of DNA-regulatory proteins, the trace element zinc plays a crucial role in the human organism. Systemic zinc deficiency becomes manifest with the triad of dermatitis, alopecia, and diarrhea. Eczematous skin lesions evolve predominantly on the extremities and periorificially with a bullous, desquamative, or vesicular aspect. Decreased activity of zinc-dependent enzymes such as alkaline phosphatase may also give rise to the suspicion of AE. Symptoms of advanced AE may include neuropsychiatric abnormalities, hypogonadism, growth retardation, and dysfunction of the immune system. Untreated AE may ultimately lead to multiple organ failure and death. ${ }^{3,4}$ In individuals with the typical clinical picture, decreased zinc plasma levels $(<0.5 \mathrm{mg} / \mathrm{L})$ corroborate the diagnosis of AE. ${ }^{5}$ Oral replacement therapy with elemental zinc at doses of 1 to $3 \mathrm{mg} / \mathrm{kg} / \mathrm{d}$ alleviates symptoms of $\mathrm{AE}$ within days to weeks, and a normal development can be expected. Zinc supplementation should be maintained throughout life; dosage may need to be increased in puberty or during pregnancy. ${ }^{3}$

\section{Participants and Methods}

We report on 5 children with AE (4 male, 1 female) who were referred to a specialist because of skin blistering. Skin punch biopsy samples were investigated histologically and by immunofluorescence analysis. In 4 of the patients, the clinical diagnosis of AE could be confirmed by detection of a mutation in SLC39A4 (Sanger sequencing data).

\section{Results}

Patient 1, now a 3-year-old boy, was referred to us at the age of 8 months with generalized epidermal blistering and loose stools. The parents had first noticed lesions of the oral mucosa and a skin rash at the age of 6 months shortly after the introduction of solid foods. Small red papules had occurred on the feet and expanded to the other extremities, the posterior scalp, and the diaper area. Some of these lesions developed into blisters.

Physical examination showed an infant in good general health with disseminated vesiculobullous skin lesions and erosions (Figure 1A), some already crusted or superinfected. Immunofluorescence staining of skin sections indicated cleavage at the basement membrane level, with complete detachment of the epidermal layers. All protein markers at the dermoepidermal junction, however, could be detected. Determination of plasma zinc and ceruloplasmin concentrations led to the diagnosis of AE: both were remarkably diminished $(0.18 \mathrm{mg} / \mathrm{L}$ and $0.21 \mathrm{~g} / \mathrm{L}$, respectively). Sequence analysis of SLC39A4 revealed compound heterozygosity for the null mutations c. $1203 \mathrm{G}>\mathrm{A}$ (p.W401X) and c.1465_1474+4del14, which have already been described as causes of severe AE. ${ }^{6,7}$ Oral zinc replacement therapy was initiated and monitored. Supplementation of $25 \mathrm{mg}$ elemental zinc daily $(2.7 \mathrm{mg} / \mathrm{kg} / \mathrm{d})$ proved sufficient. Under this regimen, the plasma zinc concentration normalized and all symptoms disappeared (Figure 1B). At the age of 21 months, zinc dosage was raised to $50 \mathrm{mg}$ elemental zinc daily $(3.5 \mathrm{mg} / \mathrm{kg} / \mathrm{d})$ because of a decreasing plasma zinc level and a known higher demand during infancy. Then, 7 months later, the plasma zinc concentration exceeded the upper limit, so replacement therapy was adjusted to $25 \mathrm{mg}$ oral zinc daily $(1.62 \mathrm{mg} / \mathrm{kg} / \mathrm{d})$. Within 8

\footnotetext{
'University Hospital Erlangen, Erlangen, Germany

${ }^{2}$ University of Freiburg, Freiburg, Germany

${ }^{3}$ University Hospital Frankfurt, Frankfurt am Main, Germany

${ }^{4}$ Vivantes Hospital Neukölln, Department of Pediatrics, Berlin, Germany

${ }^{5}$ Center for Human Genetics Freiburg, Germany
}

\section{Corresponding Author:}

Johanna Hammersen, Department of Pediatrics, University Hospital Erlangen, Loschgestr 15, 91054 Erlangen, Germany.

Email: johanna.hammersen@uk-erlangen.de 


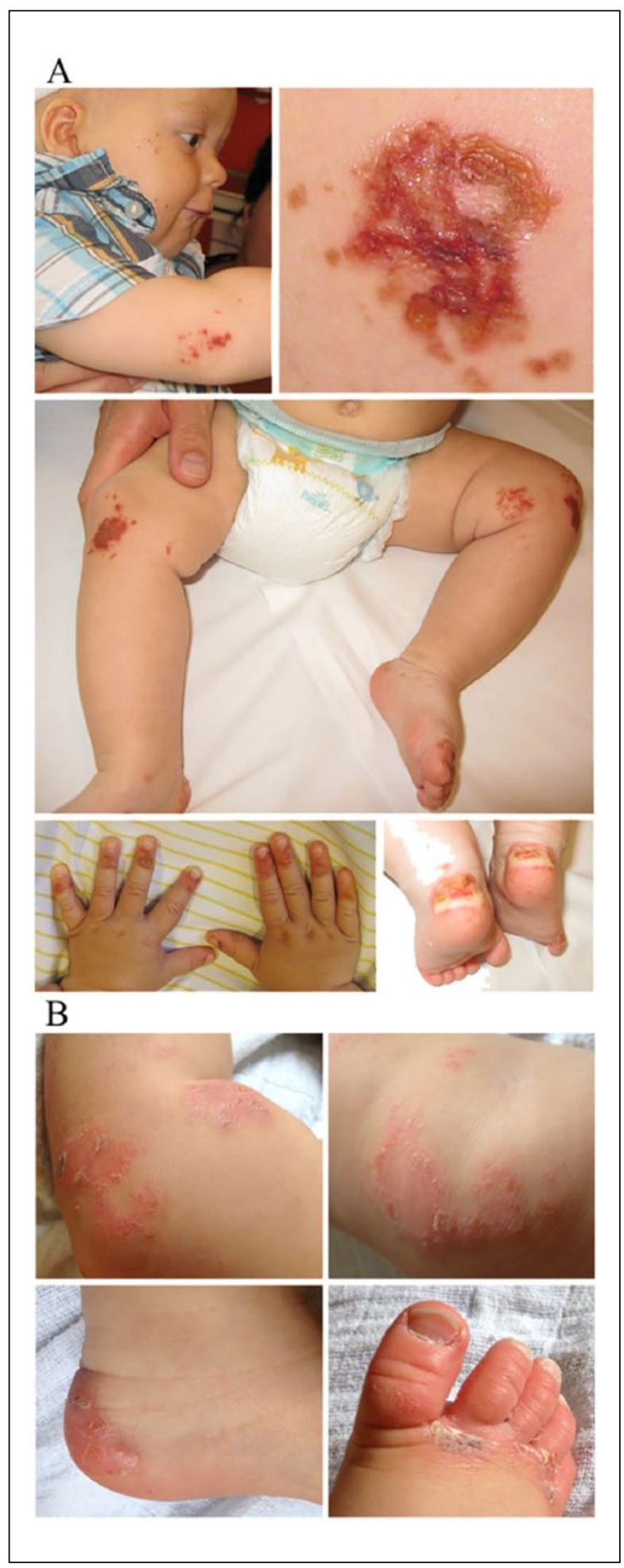

Figure I. A. Photographs displaying various vesiculobullous skin lesions at the extremities, the face, and the neck of an 8-month-old boy with AE (patient I) before zinc supplementation was started. B. Healing skin lesions at the elbows, the right heel, and toes 2 weeks after initiation of oral zinc replacement therapy. weeks, the plasma zinc level dropped below the normal range (Figure 2) and symptoms recurred. Zinc supplementation of 2 to $3 \mathrm{mg} / \mathrm{kg} / \mathrm{d}$ was resumed and led to quick disappearance of all symptoms.

Patient 2, another now 3-year-old boy of Asian origin, was seen in a genodermatoses clinic at the age of 4 months because of perioral and acral skin blistering combined with diarrhea 1 month after weaning from breast milk. Oral zinc supplementation was started, and the symptoms ameliorated quickly. Unfortunately, the clinical course could not be followed because the family left the country.

Patient 3, now a 5-year-old boy, also presented with perioral and acral blisters a few weeks after introduction of solid foods. His plasma zinc concentration was 0.10 $\mathrm{mg} / \mathrm{L}$, reflected by a reduced activity of alkaline phosphatase (32 U/L). After initiation of zinc supplementation $(3 \mathrm{mg} / \mathrm{kg} / \mathrm{d})$, the diagnosis of AE was confirmed by detection of compound heterozygosity for 2 known SLC39A4 mutations. With regular adjustment of zinc dosage to the increasing body weight, a relevant decline in the patient's plasma zinc level (to $0.18 \mathrm{mg} / \mathrm{L}$, associated with temporary recurrence of skin lesions) has been observed only once.

Patient 4, now a 4-year-old girl, and patient 5, her younger brother, were siblings with consanguineous parents of Middle-Eastern origin and a family history of a fatal skin disorder: 2 of their uncles had died in early infancy as a result of severe skin blistering.

Both patients were breastfed until the age of 8 weeks. Soon after weaning they developed diarrhea, recurrent perioral, enoral, and perianal skin erosions as well as skin blistering of hands and feet. When patient 4 was 6 months old, oral zinc supplementation as tentative treatment led to a remarkable alleviation of all symptoms. Patient 5 also received low-dose oral zinc supplementation when he was referred to us at the age of 6 months with a suspected diagnosis of epidermolysis bullosa (EB). We saw a 6-month-old male infant in good general health with perioral and perinasal skin erosions as well as blisters on the hands and feet (Figure 3A). Immunofluorescence analysis of a skin biopsy sample showed no abnormality. All protein markers at the dermoepidermal junction were detectable. Histological assessment, however, revealed acanthosis, elongated dermal papillae, parakeratosis, and Munro's microabscesses (Figure 3B). The zinc serum level was diminished $(0.38 \mathrm{mg} / \mathrm{L})$. In both siblings, sequence analysis of SLC39A4 confirmed the suspected diagnosis of AE by disclosing homozygosity for the previously undescribed mutation c.71delC (p.P24Rfs*6). This mutation creates a premature termination codon. Oral zinc supplementation in patients 4 and 5 was continued at higher doses, resulting in 


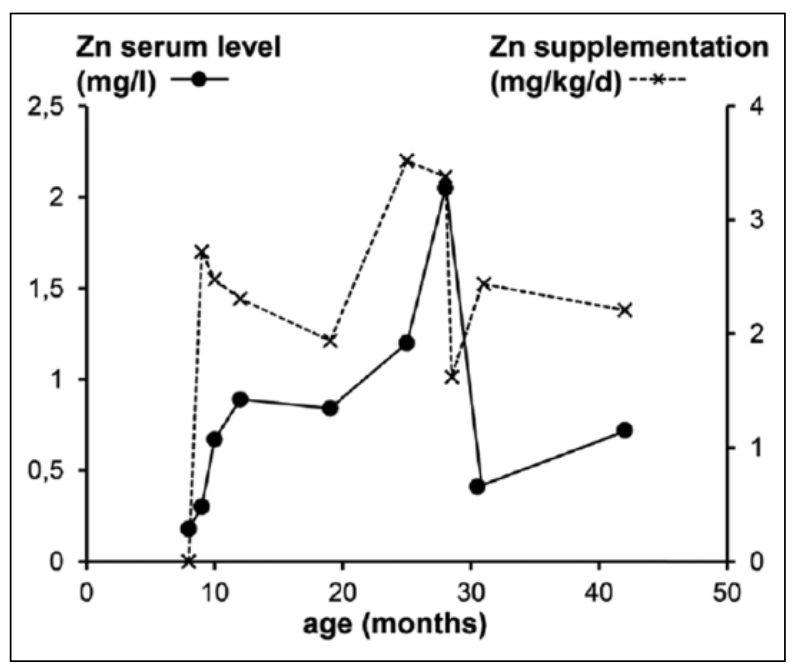

Figure 2. Graphs displaying a strong correlation of the course of zinc plasma levels of patient I (continuous line with filled circles, left-side scale on the $y$-axis) with the oral zinc dose administered (dashed line with crosses, right-side scale on the $y$-axis) throughout infancy.

increased plasma zinc concentrations and disappearance of all symptoms. Interestingly, $0.9 \mathrm{mg} / \mathrm{kg} / \mathrm{d}$ proved sufficient in these siblings. Patient 4 did not show any signs of AE when her plasma zinc level was only $0.42 \mathrm{mg} / \mathrm{L}$. After a treatment-free interval of 10 days, however, both siblings developed perioral erosions, which disappeared once the zinc supplementation was resumed.

\section{Discussion}

In all $\mathrm{AE}$ patients reported here, vesiculobullous skin lesions were the reason to seek medical help. The lesions occurred soon after weaning from breast milk or introduction of solid foods, at ages typical for the primary manifestation of AE. ${ }^{3}$ Because clinical manifestation of $\mathrm{AE}$ correlates with weaning from breast milk, both food allergies and food intolerances are important differential diagnoses: cow's milk protein allergy may cause AE-like skin lesions, ${ }^{8}$ whereas other food allergies can lead to acquired zinc deficiency and, thus, to a similar clinical picture. ${ }^{9}$ A bullous phenotype may also be suggestive of hereditary EB, a group of genodermatoses in which defective anchoring proteins at the dermoepidermal junction cause fragility of the skin and mucous membranes. ${ }^{10}$ Skin blistering caused by AE has been reported repeatedly and may mislead clinicians in the first place. ${ }^{11,12}$

The fact that 2 untreated relatives of the patients reported here had died in their second year of life and

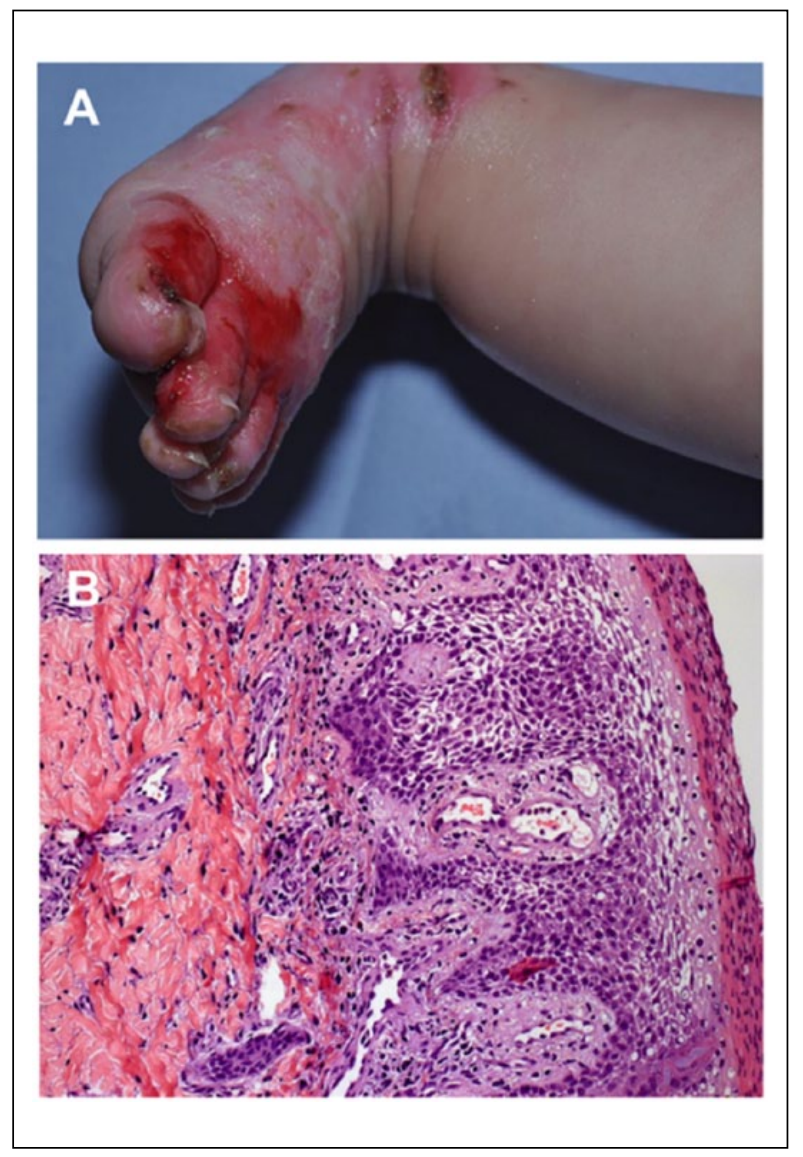

Figure 3. A. Photographs of vesiculobullous skin lesions at the foot of a 6-month-old boy with AE (patient 5). B. Hematoxylin-eosin-stained section of a skin biopsy specimen from this patient displaying papillomatosis, parakeratosis, and lymphohistiocytic infiltrates in the upper dermis.

the clinical courses with complete disappearance of symptoms after initiation of oral zinc supplementation and recurrence when the dose was reduced or treatment was discontinued underline the importance of a highdose zinc supplementation throughout infancy. A plasma zinc concentration of $0.5 \mathrm{mg} / \mathrm{L}$ appeared to be a cutoff value for clinical symptoms of zinc deficiency in a previous study, with a specificity of $89 \% .{ }^{13}$ At a plasma zinc concentration of $0.41 \mathrm{mg} / \mathrm{L}, 1$ of our patients redeveloped symptoms of $\mathrm{AE}$, whereas another one was asymptomatic at $0.42 \mathrm{mg} / \mathrm{L}$, indicating that this parameter at a distinct moment does not necessarily predict the phenotype of hereditary zinc deficiency. It may vary intraindividually and interindividually depending on the duration of zinc depletion or the severity of $\mathrm{AE} .{ }^{13}$ Nevertheless, the clinical courses reported here confirm the need for weight-adapted zinc supplementation throughout infancy. 
In summary, this study shows that $\mathrm{AE}$ is an important differential diagnosis of genetic blistering disorders in infants, especially when skin lesions are found periorificially and the onset of symptoms correlates with the introduction of solid foods. Once the diagnosis of AE is established, high-dose zinc supplementation must be retained.

\section{Acknowledgments}

We are very grateful to the patients and their families as well as to all members of the medical teams involved in their care. We thank Dr Agnes Schwieger-Briel (Kinderspital Zürich) and Dr Michael Goroncy (Taunusstein) for clinical advice and Dr Kristin Technau-Hafsi (Department of Dermatology, Medical Center, University of Freiburg) for histological analysis of some of the patients' punch biopsies. This research was not supported by any specific grant from a funding agency in the public, commercial, or not-for-profit sectors.

\section{Author Contributions}

$\mathrm{JH}, \mathrm{CH}, \mathrm{MG}, \mathrm{ML}, \mathrm{RR}$, and HS contributed to the collection of clinical data, data analysis and interpretation. $\mathrm{CH}$ performed immunofluorescence analyses and histological assessments. JK provided molecular genetic data. JH and HS wrote the manuscript.

\section{Declaration of Conflicting Interests}

The author(s) declared no potential conflicts of interest with respect to the research, authorship, and/or publication of this article.

\section{Funding}

The author(s) received no financial support for the research, authorship, and/or publication of this article.

\section{References}

1. Küry S, Kharfi M, Blouin E, Schmitt S, Bézieau S. Clinical utility gene card for: acrodermatitis enteropathica - update 2015. Eur J Hum Genet. 2016;24(5). doi:10.1038/ejhg.2015.203.
2. Wang K, Zhou B, Kuo YM, Zemansky J, Gitschier J. A novel member of a zinc transporter family is defective in acrodermatitis enteropathica. Am J Hum Genet. 2002;71:66-73.

3. Maverakis E, Fung MA, Lynch PJ, et al. Acrodermatitis enteropathica and an overview of zinc metabolism. $J \mathrm{Am}$ Acad Dermatol. 2007;56:116-124.

4. Perafán-Riveros C, França LF, Alves AC, Sanches JA Jr. Acrodermatitis enteropathica: case report and review of the literature. Pediatr Dermatol. 2002;19:426-431.

5. Schmitt S, Küry S, Giraud M, Dréno B, Kharfi M, Bézieau S. An update on mutations of the SLC39A4 gene in acrodermatitis enteropathica. Hum Mutat. 2009;30: 926-933.

6. Jung AG, Mathony UA, Behre B, et al. Acrodermatitis enteropathica: an uncommon differential diagnosis in childhood-first description of a new sequence variant. $J$ Dtsch Dermatol Ges. 2011;9:999-1002.

7. Küry S, Kharfi M, Kamoun R, et al. Mutation spectrum of human SLC39A4 in a panel of patients with acrodermatitis enteropathica. Hum Mutat. 2003;22:337-338.

8. Solomon J, Kamalammal R, Sait MY, Lohith H. Cow's milk protein allergy mimicking acrodermatitis enteropathica. J Clin Diagn Res. 2014;8:160-161.

9. Martin DP, Tangsinmankong N, Sleasman JW, DayGood NK, Wongchantara DR. Acrodermatitis enteropathica-like eruption and food allergy. Ann Allergy Asthma Immunol. 2005;94:398-401.

10. Fine JD. Inherited epidermolysis bullosa. Orphanet $J$ Rare Dis. 2010;5:12.

11. Coromilas A, Brandling-Bennett HA, Morel KD, Chung WK. Novel SLC39A4 mutation in acrodermatitis enteropathica. Pediatr Dermatol. 2011;28:697-700.

12. Jensen SL, McCuaig C, Zembowicz A, Hurt MA. Bullous lesions in acrodermatitis enteropathica delaying diagnosis of zinc deficiency: a report of two cases and review of the literature. J Cutan Pathol. 2008;35(suppl 1):1-13.

13. Wessells KR, King JC, Brown KH. Development of a plasma zinc concentration cutoff to identify individuals with severe zinc deficiency based on results from adults undergoing experimental severe dietary zinc restriction and individuals with acrodermatitis enteropathica. J Nutr. 2014;144:1204-1210. 